\title{
Evaluation of a glycerol-preserved antigen in the direct agglutination test for diagnosis of visceral leishmaniasis at rural level in eastern Sudan
}

Correspondence

Abdallah el Harith

harith17@yahoo.com

Received 1 June 2006

Accepted 1 July 2006

\author{
Mohamed el Mutasim, Durria Mansour, Elfadil M. Abass, Wisam M. Hassan \\ and Abdallah el Haritht
}

Ahfad University for Women, PO Box 167, Omdurman, Sudan

\section{INTRODUCTION}

Over the past two decades, significant progress has been achieved in the diagnosis of visceral leishmaniasis (VL). Antibody- and antigen-based detection techniques have been developed and assessed to replace the less-sensitive and invasive parasitological methods. Evaluation of the available results indicate that the direct agglutination test (DAT), urine latex agglutination and the recombinant antigen (rK39) strip/ dipstick test are adequately reliable and, in combination with clinical manifestations typifying the disease, could replace parasitological techniques (el Harith et al., 1988; Burns et al., 1993; Attar et al., 2001). The scarce or irregular availability of these diagnostic tools in the affected areas themselves, however, remains a problem. Among other factors, the

tPresent address: Wijngaard 155, 8212 CJ Lelystad, The Netherlands.

Abbreviations: DAT, direct agglutination test; FD, freeze-dried; FF, formaldehyde-fixed; GP, glycerol-preserved; ROC, receiver-operating characteristics; VL, visceral leishmaniasis. accessibility of patients and physicians to these diagnostics in developing countries depends on financial, logistical and sometimes political factors. Production of these diagnostic tools locally will therefore contribute significantly to minimizing fatalities in numbers such as those witnessed in the southern region of Sudan (de Beer et al., 1991).

In comparison with the rK39 strip/dipstick and urine latex agglutination, the DAT appears to be the simplest technique for reproducible results, as identification and production of unique antigens or antibodies requires sophisticated equipment and a certain level of skill. Thus, local DAT production has been achieved satisfactorily by a number of research groups in endemic areas (Pal et al., 1991; Mbati et al., 1999; Bimal et al., 2005; Singh et al., 2005).

To maintain DAT stability in adverse temperatures, freezedrying of the antigen was introduced to test procedures (Meredith et al., 1995). However, the application of freezedrying to fragile promastigotes is difficult to achieve 
successfully under laboratory conditions in developing countries. A simple and economical alternative method using glycerol as a thermo-protectant has therefore been developed and evaluated against a commercialized DAT freeze-dried kit at the laboratory level with favourable results (el Harith et al., 2003).

The objective of this study was to determine more realistically the stability of the glycerol-preserved (GP) antigen under ambient conditions in one of the health settings in Gedaref state, a major VL-endemic area in eastern Sudan.

\section{METHODS}

Study area and population. The study was conducted in Doka, a small town of 50 000-60000 inhabitants located $\sim 50 \mathrm{~km}$ south-east of El Gedaref, the state capital, and a similar distance from the Sudanese border with Ethiopia. The ambient temperature declines to $28^{\circ} \mathrm{C}$ from October to February, but fluctuates between 35 and $47^{\circ} \mathrm{C}$ from March to November.

Compared with hospitals in the Sudanese Eastern province, the existing hospital in Doka is considered to be of moderate capacity (50-60 beds). Among other essential facilities, the laboratory attached to the hospital is equipped with a refrigerator operating on rationed (9-11 $\mathrm{h}$ per day) electric power and a solar-energy-powered freezer $\left(-20^{\circ} \mathrm{C}\right)$.

The inhabitants residing in Doka area are well aware of 'kala-azar' or VL, the symptoms of the disease and the subsequent risks if the disease is left untreated. Patients who develop fever for 2 or more weeks and do not respond to anti-malarial drugs are therefore less hesitant in reporting to the peripheral hospital. These patients are additionally encouraged by the knowledge that diagnosis is becoming less invasive and less painful and that treatment is free of charge. On average, 5-20 patients with symptoms resembling VL are received daily at Doka hospital.

Our study was facilitated by an ongoing VL epidemiological project undertaken by the Institute of Endemic Diseases, University of Khartoum, in the same area. By coordination with the Institute of Endemic Diseases and organizing orientation meetings with health authorities in Gedaref state on the nature and objectives of our study, the necessary permission was granted by the kala-azar National Control Programme Coordinator. Due to ethical and financial restrictions, we were not able to perform prior human immunodeficiency virus/AIDS testing on suspected VL cases enrolled in the study.

From September 2004 to October 2005, six visits of 2-4 weeks duration were made by our team to Doka peripheral hospital.

Enrolment of patients. Of 308 suspected VL patients received at the peripheral hospital, 204 were males and 104 females. Their ages ranged from 3 to 40 years. Almost all patients manifested spleen and/or lymph node enlargement in addition to fever. Patients and/or their guardians were informed about the possibility of VL and how the diagnostic procedures would be performed. With the patient's consent, $5 \mathrm{ml}$ venous blood was collected, followed by aspiration of one or both inguinal lymph nodes. Patients with negative Giemsastained smears (read independently by at least two observers) in the first examination were subjected to a second or third lymph node aspiration. With the exception of six patients diagnosed either by bone marrow (3) or spleen (3) aspiration at the central hospital in Gedaref, the remaining 302 patients were assessed by the above protocol.

Sera were separated from the blood samples using a manually operated centrifuge and each sample was divided into two portions for direct testing with DAT at Doka peripheral hospital or freezing at $-20{ }^{\circ} \mathrm{C}$ (solar-powered freezer) until cold-chain transportation to the central laboratory in Omdurman.

Patients with positive lymph node, bone marrow or spleen aspirates or with positive VL clinical presentation and a DAT titre of $\geqslant 1: 3200$ (el Harith et al., 1988) were put on a 30 day treatment course with sodium stibogluconate (Pentostam; Albet-Davis SSG). All other patients who were assessed to be negative for VL were advised to seek further medical care in the state central hospital in Gedaref.

\section{Preparation of DAT antigens}

Formaldehyde-fixed (FF) antigen. Promastigotes of Leishmania donovani (strain MHOM/68/1-S; an isolate from a VL case in Sudan) were mass cultured in liver infusion/tryptose medium supplemented with $10 \%$ fetal calf serum and processed according to the improved DAT procedures described previously (el Harith et al., 1995). The stained promastigote suspension was washed and finally resuspended in $1.2 \%(\mathrm{v} / \mathrm{v})$ formaldehyde/citrate saline as $10 \times$ concentrated stock antigen $\left(5 \times 10^{8}\right.$ promastigotes $\left.\mathrm{ml}^{-1}\right)$.

Following a similar protocol, 17 valid DAT antigen batches varying in volume from 176 to $3200 \mathrm{ml}$ (sufficient for testing of 290 to 5300 patients, respectively) were successfully produced in our laboratory over the period 1999-2005 (el Harith et al., 2002; and unpublished data). The reliability of this in-house-produced antigen (including the current study) was evaluated against standard control sera from eight healthy subjects and eight confirmed VL patients.

For this study, two stock FF antigen suspensions of $80 \mathrm{ml}$ each $\left(5 \times 10^{8}\right.$ promastigotes $\mathrm{ml}^{-1}$ ) were prepared according to the standard procedures described above and stored at $4{ }^{\circ} \mathrm{C}$ in the central laboratory in Omdurman until required.

Glycerol-preserved (GP) antigen. The procedures followed for DAT antigen preservation were as described by el Harith et al. (2003) except that the glycerol concentration was increased to $75 \%$ $(\mathrm{v} / \mathrm{v})$. A glycerol concentration of $75 \%$ was used in this study following favourable results obtained at a challenging temperature of $50{ }^{\circ} \mathrm{C}$ for at least 52 days (unpublished data).

Eighty millilitres of the final FF antigen was centrifuged $(3200 \mathrm{~g}$ ) for $10 \mathrm{~min}$ at $4{ }^{\circ} \mathrm{C}$ and resuspended in $20 \mathrm{ml} 1 \cdot 2 \%(\mathrm{v} / \mathrm{v})$ formaldehyde/ citrate saline to which $60 \mathrm{ml}$ glycerol (propane-1,2,3-triol; Philip Harris) was added. The antigen/protectant suspension $(10 \times$ concentrated stock antigen) was mixed thoroughly and divided into $1.8 \mathrm{ml}$ aliquots. Twenty of the aliquots were transported (without cooling) in September 2004 to Doka peripheral hospital where they were kept at ambient laboratory temperature $\left(28-47^{\circ} \mathrm{C}\right)$ throughout the study period (13 months). Another 20 aliquots were kept at ambient temperature at the central laboratory in Omdurman $\left(25-37^{\circ} \mathrm{C}\right)$ with or without air-conditioning.

Freeze-dried (FD) antigen. A total of 110 vials of $5 \mathrm{ml} \mathrm{FD}$ antigen (lot no. 1001) were purchased via the East Mediterranean Regional Office of the World Health Organization in Cairo. Following the instructions provided, the FD antigen was stored at ambient temperature in the central laboratory in Omdurman (with or without air-conditioning: $\left.25-37^{\circ} \mathrm{C}\right)$ or in the peripheral hospital in Doka $\left(28-47^{\circ} \mathrm{C}\right)$.

By applying a similar diagnostic dose $(0.6 \mathrm{ml}$ per test $)$ to the in-house GP- and FF-produced antigen versions, the cost of using the FD kit was US $\$ 2.96$ per patient.

On arrival at the peripheral hospital in Doka, reconstituted samples from the GP, FD and FF antigens were examined microscopically to ensure the absence of promastigote clump formation (autoagglutination) or lysis due to transportation. 
DAT. One day before the DAT was carried out, the GP and FF stock suspensions were reconstituted by the addition of nine equal volumes of $1.2 \%(\mathrm{v} / \mathrm{v})$ formaldehyde/citrate saline (el Harith et al., 2003). These reconstituted antigen aliquots were left overnight at ambient temperature (GP) or at $4-8{ }^{\circ} \mathrm{C}(\mathrm{FF})$. Reconstitution of the FD antigen was carried out according to the instructions provided. Five millilitres of physiological saline $(0.9 \% \mathrm{NaCl})$ was added to each vial 5-10 min prior to the DAT, followed by occasional gentle mixing. Similar test procedures were followed in the central laboratory in Omdurman. Gelatin was used as a serum diluent for the GP and FF antigen preparations (el Harith et al., 1995). For the FD antigen, the serum diluent was prepared according to the instructions provided (Meredith et al., 1995).

Standard positive and negative serum samples were included in each DAT. Irrespective of the antigen version used, twofold dilutions of sera were tested from a dilution of $1: 100$ up to $1: 102400$. After addition of the respective antigens, microtitre plates were shaken manually for 1-2 min. The development of a reaction was observed at $\sim 1 \mathrm{~h}$ intervals during the day. Following overnight incubation at ambient temperatures (health setting: $28-47^{\circ} \mathrm{C}$; central laboratory: $25-37^{\circ} \mathrm{C}$ ), test results were read visually (against a white background) by at least two observers. The end point was estimated by localizing a clear sharpedged blue spot (in contrast to a clear colourless supernatant) identical to that observed in the control well; the preceding dilution was taken as the titre of the serum sample. Patients showing titres of $\geqslant 1: 3200$ were considered to be VL (sero)positive (el Harith et al., 1988).

Data analysis. If normally distributed, numerical variables were calculated as means $\pm \mathrm{SD}$; otherwise, they were calculated as median and quartile values. Mean values were compared using Student's $t$ test and medians using the Mann-Whitney U-test, at a critical $\alpha$ level of 0.05 ; all tests were two-tailed. The reproducibility of the GP, FD and FF result values obtained in the peripheral hospital and the central laboratory was assessed by Cohen's kappa. The sensitivity and specificity of the GP, FD and FF antigens were estimated as percentages of the true (parasitologically confirmed) positives or negatives with exact binomial $95 \%$ confidence limits. To determine the ideal DAT cut-off value for the three antigens in the peripheral hospital and central laboratory, a receiver-operating characteristics (ROC) curve was constructed (Fletcher et al., 1982). For comparison of differences among the diagnostic results obtained with the three antigen preparations, a Bland-Altman plot was applied. Data were analysed with the STATA software program for Windows.

The small size of the study population and the high correlation between variables did not allow a valid latent class analysis to be carried out.

\section{RESULTS}

Despite transportation without a cold-chain facility, no abnormalities were observed in promastigote morphology nor was there a tendency for sedimentation to occur in the reconstituted GP antigen, implying the absence of autoagglutination. As with the FF preparation, but not the FD preparation, provisional negative results (spot formation) could therefore be visualized for the GP antigen preparation within 2-3 h of incubation. The formation of a diffuse blue background occurred with the FD antigen preparation, rendering localization of the end-point reaction somewhat difficult.

Of 302 suspected VL cases examined in Doka peripheral hospital and six in Gedaref central hospital, $174(56 \cdot 5 \%)$ had negative organ aspirates. Regardless of the antigen preparation used, all 174 also scored unambiguous negative DAT titres $(1: 100-1: 400)$ in the peripheral hospital, as well as in the central laboratory. The results obtained in 87 $(28.2 \%)$ patients who tested positive in the DAT (titres of $1: 3200-1: 102400)$ against the GP, FD and FF in both the peripheral and the central laboratory settings were also in agreement with the positive organ aspirate results. Despite this high degree of concordance $(261 / 308,84 \cdot 7 \%)$, some discrepancy between the results of organ aspiration and the DAT results was observed in $18(5 \cdot 8 \%)$ other patients. Eight of these had a positive organ aspirate (seven lymph nodes and one spleen) but a negative $(1: 100-1: 400)$ or marginally negative $(1: 800-1: 1600)$ DAT reading against all three antigens in the two settings. Despite negative lymph node aspirate results in the remaining ten patients, positive DAT readings $(1: 3200-1: 102400)$ were recorded for all three antigen preparations. For the remaining 29 patients (9.4\%), concordance or discrepancy between the results of the DAT and organ aspiration was dependent on the individual antigen preparation used and/or the health setting.

No significant difference was observed between the mean titres obtained with the GP and FD antigen preparations in the peripheral hospital $(P=0.945)$ or in the central laboratory $(P=0 \cdot 074)$. However, there was a slight but significant difference between the FF and GP antigen preparation mean titres in both the peripheral hospital $(P=0 \cdot 019)$ and the central laboratory $(P=0 \cdot 000)$; four $(1 \cdot 3 \%)$ patients tested negative with the GP antigen but positive with the FF antigen in both settings. Despite this difference, DAT readings obtained with the three antigens in the vast majority of patients $(304 / 308,98.7 \%)$ showed excellent agreement between antigen preparations at both the peripheral hospital and the central laboratory (weighted kappa $>0 \cdot 90$ ). When parasitology was taken as the gold standard and a titre of $1: 3200$ was taken as the cut-off point, comparable sensitivities of $86 \cdot 7,87 \cdot 6$ and $89 \cdot 5 \%$ were obtained with the GP, FD and FF antigen preparations, respectively, in the peripheral hospital (Table 1). At this cutoff value, specificities of $92 \cdot 1,92 \cdot 6$ or $92 \cdot 6 \%$, respectively, were obtained in the patient group with negative lymph node aspirate results. At the lower but optimal cut-off point established by ROC analysis $(1: 400-1: 800)$, higher sensitivity levels $(90 \cdot 5-96 \cdot 2 \%)$ were estimated for all three antigen preparations. Similar high levels of specificity $(94 \cdot 6-96 \cdot 5 \%)$ could be achieved if the ten patients who were misdiagnosed by lymph node aspiration (as a result of clinical presentation, positive DAT readings and a favourable response to chemotherapy) were classified as genuine VL cases.

All 115 VL cases, whether diagnosed by organ aspiration (105) or on the basis of clinical manifestation and a positive DAT outcome (10), were put on a 30 day treatment course with sodium stibogluconate following the guidelines of the Ministry of Health and WHO (2004). All 115 patients diagnosed and treated in this way showed a favourable response. 
Table 1. Efficiency of GP, FD and FF antigen preparations for diagnosis of VL at a pre-established DAT cut-off value

Sensitivity is defined as the number (percentage) of DAT-positive cases from 105 cases positive by organ aspirate [lymph node (99), bone marrow (3) and spleen (3)]. The DAT cut-off value for VL was $1: 3200$. Specificity is defined as the number (percentage) of DAT-positive cases from 203 cases negative by lymph node aspirate. CI, Confidence interval.

\begin{tabular}{|lcc|}
\hline Antigen variant/location & Sensitivity (\%) [exact 95\% CI] & Specificity (\%) [exact 95\% CI] \\
\hline GP & $91(86 \cdot 7 \%)[78 \cdot 6-92 \cdot 5]$ & $187(92 \cdot 1 \%)[86 \cdot 3-94 \cdot 7]$ \\
Peripheral hospital & $92(87 \cdot 6 \%)[80 \cdot 7-93 \cdot 9]$ & $185(91 \cdot 1 \%)[87 \cdot 5-95 \cdot 4]$ \\
Central laboratory & $92(87 \cdot 6 \%)[79 \cdot 8-93 \cdot 2]$ & $188(92 \cdot 6 \%)[88 \cdot 1-95 \cdot 8]$ \\
FD & $92(87 \cdot 6 \%)[79 \cdot 8-93 \cdot 2]$ & $187(92 \cdot 1 \%)[86 \cdot 3-94 \cdot 7]$ \\
Peripheral hospital & & \\
Central laboratory & $94(89 \cdot 5 \%)[82 \cdot 0-94 \cdot 7]$ & $188(92 \cdot 6 \%)[88 \cdot 1-95 \cdot 8]$ \\
FF & $94(89 \cdot 5 \%)[82 \cdot 0-94 \cdot 7]$ & $185(91 \cdot 1 \%)[87 \cdot 5-95 \cdot 4]$ \\
Peripheral hospital & & \\
Central laboratory & &
\end{tabular}

\section{DISCUSSION}

The development of practical diagnostic methods such as the DAT are among the recommendations of the WHO Special Programme for Research and Training in Tropical Diseases to strengthen capacities for VL control in developing countries (WHO, 2004). In our opinion, both local production and sustaining diagnostic reliability of these tools under rural conditions are equally crucial. Owing to improvements that have been introduced, processing and maintaining the stability of DAT antigens have been adequately simplified to cope with the less-than-optimal conditions in VL-endemic areas (el Harith et al., 1988, 1995, 1996, 2003). Making use of these simplifications, several valid DAT FF and GP antigen batches have been produced in our laboratory, ensuring the long-term availability of the test at a reduced cost of US $\$ 0 \cdot 40-0 \cdot 45$ per patient (compared with approximately US\$3.00 for the imported FD antigen kit).

In agreement with observations in previous studies where the DAT antigen (at promastigote concentrations of $5 \times 10^{7}$ or $2 \times 10^{8} \mathrm{ml}^{-1}$ ) was employed, provisional diagnostic results could be obtainable with the GP antigen within 2-3 h of incubation (el Harith et al., 1988, 2003; Schoone et al., 2001). For the FD antigen, this rapid diagnostic time could not be achieved and the appearance of a diffuse blue background in the DAT (with blurring of blue spots in negative wells) implied possible alterations in the parasite membrane or loss of the Coomassie blue stain. This may be because lowering of temperatures to the levels required for freeze-drying $(-80$ to $-85^{\circ} \mathrm{C}$ ) in the absence of cryoprotectant can be injurious and even lytic to cells (Coriell, 1979).

Even at fluctuating ambient temperatures of $28-47^{\circ} \mathrm{C}$ in the peripheral hospital, the GP antigen demonstrated a sensitivity level $(86 \cdot 7 \%)$ comparable to that of the FD antigen $(87 \cdot 6 \%)$ kept under similar conditions and with that of the FF antigen $(89 \cdot 5 \%)$ at an optimal temperature of
$4-8{ }^{\circ} \mathrm{C}$. Although this level of sensitivity seems reasonable, it is still below those reported in studies where bone marrow or spleen aspiration was employed as the gold standard (el Harith et al., 1988; Boelaert et al., 1999; Osman et al., $2000)$. In this study, lymph node aspiration missed ten $(8.7 \%)$ of the patients in whom all three antigen preparations scored positive DAT readings and in whom a favourable response to specific anti-leishmanial chemotherapy was evident. If clinical cure rather than positive parasitology was taken as the gold standard in these ten cases, slightly higher sensitivity $(87 \cdot 8,88 \cdot 7$ and $90 \cdot 4 \%)$ and much higher specificity $(96 \cdot 9,97 \cdot 4$ or $97 \cdot 4 \%)$ levels for the GP, FD or FF antigens, respectively, could be attained, even at the peripheral hospital.

A titre range of $1: 400-1: 800$ determined by ROC analysis was found to be optimal as the cut-off point for all three antigen preparations. We believe that this is a more realistic value, since, in this area of Sudan, no authentic case of family-related Trypanosoma infection has been reported so far (which can cause cross-reactivity). Based on this realistic cut-off point and taking a positive response to chemotherapy as the gold standard, diagnostic efficiencies (sensitivity and specificity $\geqslant 90 \%$ ) closely matching those obtained at the central laboratory could be reached at the peripheral hospital (el Harith et al., 1988, 1995; Boelaert et al., 1999; Osman et al., 2000). It is generally accepted that the reliability of DAT for VL detection depends to a large extent on the quality of antigen and on the area in which the VL study is carried out. When using trypsinized Leishmania promastigote antigen in an area that was equally co-endemic for trypanosomiasis, a cut-off titre of $1: 3200$ was found to be optimal (el Harith et al., 1988). However, a much lower cut-off value $(1: 400)$ could have been used in studies where the specificity of the DAT, due to 2-mercaptoethanol and urea incorporation, was remarkably enhanced, even versus Trypanosoma infections (el Harith et al., 1995). 
Failure to demonstrate Leishmania parasite, even with repeated lymph node aspiration, in $8 \cdot 7 \%$ of the genuine VL cases in this study raises the question of whether negative parasitology can rule out VL (Boelaert et al., 1999). By employing relatively more sensitive parasitological methods such as spleen aspiration and choosing a cut-off value of twice that used here $(1: 6400)$, the DAT proved to be more sensitive (Veenken et al., 2003).

The marginally negative $(1: 800-1: 1600)$ or clearly negative $(1: 100-1: 400)$ titres obtained in eight $(6.9 \%)$ of our patients with positive lymph node (7) or spleen (1) aspirates is difficult to explain. A number of immunosuppressive conditions are commonly encountered in such remote areas in Sudan, such as malnutrition and tuberculosis, which could affect the results. Other predisposing conditions such as human immunodeficiency virus/AIDS cannot be ruled out, as we were not able to assess our suspected VL cases accordingly.

In conclusion, the GP antigen preparation demonstrated a high diagnostic efficiency and test reproducibility for VL comparable to the standard FF antigen and showed stability under high ambient temperatures similar to the FD antigen. It therefore has the potential to replace both antigens for use in rural hospitals.

\section{ACKNOWLEDGEMENTS}

We wish to thank Dr Gasim Badri, President of Ahfad University, Dr Farouk A/Aziz, Dean of the School of Medicine, and Dr Abubaker Uro, Head of the Ahfad Centre for Science and Technology, for their invaluable support and encouragement. The efforts of Dr M. Mukhtar and Dr W. el Amin, Institute of Endemic Diseases, University of Khartoum, for cryopreservation and maintenance of the Leishmania strain are greatly appreciated. We are greatly indebted to $\mathrm{Dr} \mathrm{M}$. Boelaert, Prince Leopold Institute of Tropical Medicine, Antwerp, Belgium, for carrying out the statistical work. This investigation received technical and financial support (grant no. SGS04/136) from the joint WHO Eastern Mediterranean Region Office (EMRO), Division of Communicable Diseases (DCD) and the WHO Special Programme for Research and Training in Tropical Diseases (TDR): EMRO/TDR small grant scheme for operational research in tropical and other communicable diseases.

\section{REFERENCES}

Attar, Z. J., Chance, M. L., el-Safi, S., Carney, J., Azazy, A., El-Hadi, M., Dourado, C. \& Hommel, M. (2001). Latex agglutination test for the detection of urinary antigens in visceral leishmaniasis. Acta Trop 78, 11-16.

Bimal, S., Das, V. N. R., Singha, P. K. \& 7 other authors (2005). Usefulness of the direct agglutination test in the early detection of subclinical Leishmania donovani infection: a community study. Ann Trop Med Parasitol 99, 743-749.

Boelaert, M., el Safi, S., Jacquet, D., de Muynck, A., van der Stuyft, P. \& Le Ray, D. (1999). Operational validation of the direct agglutination test for diagnosis of visceral leishmaniasis. Am J Trop Med Hyg 60, 129-134.

Burns, J. M., Jr, Shreffler, W. G., Benson, D. R., Ghalib, H. W., Badaro, R. \& Reed, S. G. (1993). Molecular characterization of a kinesin-related antigen of Leishmania chagasi that detects specific antibody in African and American visceral leishmaniasis. Proc Natl Acad Sci U S A 90, 775-779.

Coriell, L. L. (1979). Preservation, storage, and shipment. Methods Enzymol 58, 29-36.

de Beer, P., el Harith, A., Deng, L. L., Semiao-Santos, S. J., Chantal, B. \& van Grootheest, M. (1991). A killing disease epidemic among displaced Sudanese population identified as visceral leishmaniasis. Am J Trop Med Hyg 44, 283-289.

el Harith, A., Kolk, A. H. J., Leeuwenburg, J., Muigai, R., Huigen, E., Jelsma, T. \& Kager, P. A. (1988). Improvement of a direct agglutination test for field studies of visceral leishmaniasis. J Clin Microbiol 26, 1321-1325.

el Harith, A., Chowdhury, S., Al-Masum, M., Semiao-Santos, S. J., Karim, E., el-Safi, S. \& Haque, I. (1995). Evaluation of cleaving agents other than trypsin in direct agglutination test for further improving diagnosis of visceral leishmaniasis. J Clin Microbiol 33, 1984-1988.

el Harith, A. E., Chowdhury, C., Al-Masum, M., Samiao-Santos, S. J., Das, P. K., Akhter, S., Vetter, J. C. M. \& Haq, I. (1996). Reactivity of various leishmanial antigens in a direct agglutination test and their value in differentiating post-kala azar dermal leishmaniasis from leprosy and other skin conditions. J Med Microbiol 44, 142-146.

el Harith, A., el Mutasim, M., Mansour, D., Mustafa, E. F., Shammat, I., Gilis, I. \& van Gool, T. (2002). National and international evaluation of in-Sudan produced direct agglutination test for visceral leishmaniasis. Sudan Med J 40, 3-11.

el Harith, A., el Mutasim, M., Mansour, D., Mustafa, E. F. \& Arvidson, H. (2003). Use of glycerol as an alternative to freeze-drying for long-term preservation of antigen for the direct agglutination test. Trop Med Int Health 8, 1025-1029.

Fletcher, R. H., Fletcher, S. W. \& Wagner, E. H. (1982). Clinical Epidemiology: the Essentials. Baltimore: Williams \& Wilkins.

Mbati, P. A., Githure, J. I., Kagai, J. M., Kirigi, G., Kibati, K., Wasunna, K. \& Koech, D. K. (1999). Evaluation of a standard direct agglutination test (DAT) for the diagnosis of visceral leishmaniasis in Kenya. Ann Trop Med Parasitol 93, 703-710.

Meredith, S. E. O., Kroon, N. C. M., Sondorp, E. \& 7 other authors (1995). Leish-KIT, a stable direct agglutination test based on freezedried antigen for serodiagnosis of visceral leishmaniasis. J Clin Microbiol 33, 1742-1745.

Osman, O. F., Kager, P. A. \& Oskam, L. (2000). Leishmaniasis in the Sudan: a literature review with emphasis on clinical aspects. Trop Med Int Health 5, 553-562.

Pal, A., Mukerji, K., Basu, D., Naskar, K., Mallick, K. K. \& Gosh, D. K. (1991). Evaluation of direct agglutination test (DAT) and ELISA for serodiagnosis of visceral leishmaniasis in India. J Clin Lab Anal 5, 303-306.

Schoone, G. J., Hailu, A., Kroon, C. C. M., Niewenhuys, J. I., Schallig, H. D. F. H. \& Oskam, L. (2001). A fast agglutination screening test (FAST) for the detection of anti-Leishmania antibodies. Trans $R$ Soc Trop Med Hyg 95, 400-401.

Singh, R., Subba Raju, B. V., Jain, R. K. \& Salotra, P. (2005). Potential of direct agglutination test based on promastigote and amastigote antigens for serodiagnosis of post-kala-azar dermal leishmaniasis. Clin Diagn Lab Immunol 12, 1191-1194.

Veenken, H., Ritmeijer, K., Seaman, J. \& Davidson, R. (2003). Comparison of an rK39 dipstick rapid test with direct agglutination test and splenic aspiration for the diagnosis of kala-azar in Sudan. Trop Med Int Health 8, 164-167.

WHO (2004). Report of the Scientific Working Group meeting on Leishmaniasis, Geneva, 2-4 February 2004. TDR/SWG/04. Geneva: World Health Organization. 